\section{Perfil de consumo de outros produtos de tabaco fumado entre estudantes de três cidades brasileiras: há motivo de preocupação?}

\author{
Smoking of non-cigarette tobacco products by \\ students in three Brazilian cities: should we be \\ worried?
}

\author{
1 Instituto Nacional de \\ Câncer, Rio de Janeiro, Brasil. \\ Correspondência \\ A. S. Szklo \\ Instituto Nacional de Câncer. \\ Rua Marquês de Pombal 125,

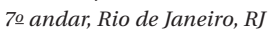 \\ 20230-240, Brasil. \\ andreszk@hotmail.com
}

\begin{abstract}
Smoking of non-cigarette tobacco products is increasing worldwide because of their high social acceptability, misperceptions about their purported harmlessness, and globalization of the tobacco industry. In Brazil, tobacco control experts have recently focused their attention on the importance of monitoring the use of such products. We analyzed data from the Global Youth Tobacco Survey (2009) in three cities. Prevalence rates of non-cigarette tobacco smoking in the previous 30 days among students 13 to 15 years of age were high in Campo Grande (18.3\%; 95\% CI: 14.4\%$22.9 \%$ ) and São Paulo (22.1\%; 95\%CI: 19.0\%25.6\%), while Vitória showed comparatively lower prevalence (4.3\%; 95\%CI: 3.1\%-5.7\%). No statistical differences were observed in prevalence rates according to gender. Water pipes were the most frequent form of non-cigarette tobacco smoking. The decline in cigarette smoking in Brazil in recent years may have contributed to other forms of tobacco smoking, especially among students.
\end{abstract}

Smoking; Consumption of Tobacco-Derived Products; Primary Prevention; Epidemiologic Surveillance

\author{
André Salem Szklo 1 \\ Mariana Miranda Autran Sampaio 1 \\ Elaine Masson Fernandes 1 \\ Liz Maria de Almeida 1
}

\section{Introdução}

A Organização Mundial da Saúde (OMS) identifica o uso do tabaco como um fator a ser controlado com alta prioridade, uma vez que é a segunda causa de morte no mundo ${ }^{1}$ e pode ser evitado. Ele está associado à mortalidade por diversos tipos de câncer, doença pulmonar obstrutiva crônica, doença coronariana, hipertensão arterial e acidente vascular encefálico 1 . Além disso, o simples fato de um indivíduo estar exposto passivamente à fumaça do tabaco, por si só já contribui para o surgimento de doenças respiratórias, do sistema cardiovascular e câncer de pulmão 1 .

Atualmente, grande parte do consumo de tabaco fumado no Brasil está relacionado ao cigarro 2 . No entanto, dada a queda que vem ocorrendo na prevalência de consumo de cigarros no país 2,3 , é importante monitorar o consumo de outros produtos derivados do tabaco, especialmente nas faixas mais jovens, em que ocorre a experimentação e/ou iniciação. Tal monitoramento precisa ir ao encontro da rapidez com que a indústria do tabaco reage às iniciativas da saúde pública 4 .

Até onde vai o conhecimento dos autores, não existe nenhum estudo publicado em revista indexada que tenha feito o levantamento do consumo de outros produtos do tabaco fumado entre adolescentes brasileiros. Apesar de existirem diversos componentes no sistema de vigilância brasileiro para o controle do tabaco $2,5,6$, foi en- 
contrado apenas um que incluiu, a partir de 2009, outros produtos de tabaco fumado tendo como público-alvo os adolescentes: o Global Youth Tobacco Survey (GYTS), conhecido no Brasil como Vigilância de Tabagismo em Escolares (Vigescola) 5,6. Essa pesquisa com escolares entre 13 e 15 anos compõe um amplo sistema de vigilância e monitoramento internacional, implementado na maioria dos Estados Membros da OMS.

O objetivo do estudo foi, portanto, avaliar a prevalência de outros produtos do tabaco fumado nas capitais brasileiras que participaram do Vigescola em 2009.

\section{Metodologia}

O Vigescola é realizado no Brasil desde 2002 5,6. A pesquisa monitora escolares entre 13 e 15 anos de idade, cursando 8o e 9o anos (antigas 7ạ e 8a séries) do Ensino Fundamental e 1a série do Ensino Médio, por meio de inquéritos repetidos e de um protocolo padronizado.

No presente artigo, foram incluídas as pesquisas realizadas nas cidades de Campo Grande (Mato Grosso do Sul), São Paulo e Vitória (Espírito Santo), em 2009 (as únicas do Vigescola a partir deste ano até o momento). A amostra de cada cidade envolveu a seleção de escolas que oferecem essas séries e de turmas, sendo elegíveis todos os alunos das turmas. Cada adolescente recebeu um questionário e o preencheu sozinho, sem se identificar. As proporções de resposta global foram 72,0\% em Campo Grande, 70,3\% em São Paulo e 81,9\% em Vitória. Participaram da pesquisa 1.018 estudantes em Campo Grande, 1.351 em São Paulo e 1.132 em Vitória.

O questionário investigou, dentre outros temas, o consumo de tabaco nos 30 dias anteriores à entrevista, distinguindo cigarros industrializados de outros produtos de tabaco fumado. Ademais, também foi avaliado o tipo de outros produtos de tabaco fumado, diferentes de cigarro industrializado, utilizados com mais frequência (cigarro de cravo/Bali, cigarro enrolado à mão, cigarrilha, charuto, fumo de mascar, narguilé ou cigarro indiano/Bidi).

Foram calculadas, segundo sexo, as prevalências de fumantes de outros produtos do tabaco e seus respectivos intervalos de 95\% de confiança, bem como a distribuição dos produtos de tabaco que foram usados com maior frequência. Utilizou-se o programa estatístico Stata 9.0 (Stata Corp., College Station, Estados Unidos) para lidar com o desenho complexo de amostragem. As "não respostas" foram levadas em consideração no cálculo dos respectivos pesos amostrais 5,6.

\section{Resultados}

As prevalências de uso de outros produtos do tabaco fumado foram de 4,3\% em Vitória, 18,3\% em Campo Grande e 21,3\% em São Paulo. Não houve diferenças estatisticamente significativas por sexo. Em Campo Grande e São Paulo, tanto entre meninos quanto entre meninas, o produto usado com maior frequência foi o narguilé (Tabela 1).

\section{Discussão}

Os achados deste artigo apontam para uma elevada prevalência de consumo de outros produtos do tabaco fumado e, em particular, de consumo de narguilé, entre os estudantes de 13 a 15 anos, de ambos os sexos, de Campo Grande e São Paulo. Esses patamares encontrados são, por exemplo, de magnitudes semelhantes às verificadas entre os jovens de alguns países do Oriente Médio e da Ásia, com larga tradição de consumo do narguilé 7,8 .

Já existem alguns estudos que mostram que o narguilé vem se espalhando pela Europa e Estados Unidos 7,8. Apesar das evidências de que seja tão nocivo para a saúde quanto o cigarro ${ }^{9}$, há uma desinformação da população geral sobre os seus danos, o que faz o uso de tal produto ser visto dentro de uma perspectiva de socialização também nos países ocidentais 7,10. As indústrias do tabaco impulsionam esse processo ao utilizarem estratégias globalizadas de disseminação de seus produtos para superar eventuais barreiras culturais e econômicas 2,4,7,8.

Vale a pena assinalar que, no Brasil, entre 1989 e 2008, foi constatado um decréscimo considerável da prevalência de fumantes de cigarros no país, em todos os estratos sociodemográficos 2,3. Tal achado reforça o fato de que ações de prevenção da iniciação e estímulo à cessação no Brasil vêm obtendo êxito com a população ${ }^{2,3}$.

No entanto, tal como observado nos resultados deste artigo, é possível que esteja havendo uma migração para outros produtos derivados do tabaco fumado diferentes do cigarro, particularmente entre os adolescentes estudantes. De fato, a prevalência de fumantes de cigarro entre estudantes de 13 a 15 anos nas capitais investigadas foi inferior (Campo Grande e São Paulo) ou equivalente (Vitória) à de outros produtos de tabaco fumado (dado não mostrado). Esse dinamismo das estratégias transnacionais da indústria do tabaco tem sempre como alvo buscar alternativas para as ações de controle do tabaco vigentes nos países e buscar novos mercados de consumidores 4,8,11. Dados recentes da Pesquisa Especial de 
Prevalência de consumo de outros produtos do tabaco fumado e distribuição de produtos usados com maior frequência entre adolescentes entre 13 e 15 anos das cidades de Campo Grande (Mato Grosso do Sul), São Paulo e Vitória (Espírito Santo). Vigescola, 2009.

\begin{tabular}{|c|c|c|c|c|c|c|c|c|c|}
\hline & \multicolumn{3}{|c|}{ Total } & \multicolumn{3}{|c|}{ Meninos } & \multicolumn{3}{|c|}{ Meninas } \\
\hline & $\mathrm{n}$ * & $\%$ & IC95\% & $n$ * & $\%$ & IC95\% & $n$ * & $\%$ & IC95\% \\
\hline \multicolumn{10}{|l|}{ Campo Grande } \\
\hline $\begin{array}{l}\text { Prevalência de consumo de outros produtos do } \\
\text { tabaco fumado }\end{array}$ & 187 & 18,3 & $14,4-22,9$ & 80 & 18,2 & $13,5-24,2$ & 107 & 18,3 & $13,8-23,8$ \\
\hline \multicolumn{10}{|l|}{ Distribuição dos outros produtos do tabaco } \\
\hline \multicolumn{10}{|l|}{ fumado usados com maior frequência } \\
\hline Cigarro de cravo/Bali & 11 & 7,7 & $3,7-15,3$ & 6 & 10,2 & $4,2-22,9$ & 5 & 5,9 & $2,8-12,2$ \\
\hline Cigarro enrolado à mão & 4 & 2,9 & $1,0-7,9$ & 1 & 1,6 & $0,2-13,0$ & 3 & 3,7 & $1,1-11,9$ \\
\hline Cigarrilha & 2 & 1,4 & $0,3-6,1$ & 2 & 3,3 & $0,7-13,8$ & 0 & - & \\
\hline Charuto, charuto pequeno & 1 & 0,7 & $0,1-5,4$ & 0 & - & & 1 & 1,3 & $0,2-8,9$ \\
\hline Narguilé & 118 & 87,3 & $79,5-92,4$ & 46 & 84,9 & $68,5-93,4$ & 72 & 89,1 & $82,8-93,2$ \\
\hline Cigarro indiano/Bidi & 0 & - & & 0 & - & & 0 & - & \\
\hline \multicolumn{10}{|l|}{ São Paulo } \\
\hline $\begin{array}{l}\text { Prevalência de consumo de outros produtos } \\
\text { do tabaco fumado }\end{array}$ & 299 & 22,1 & $19,0-25,6$ & 142 & 23,3 & $19,6-27,4$ & 157 & 21,1 & $17,4-25,4$ \\
\hline \multicolumn{10}{|l|}{ Distribuição dos outros produtos do tabaco } \\
\hline \multicolumn{10}{|l|}{ fumado usados com maior frequência } \\
\hline Cigarro de cravo/Bali & 6 & 3,0 & $1,3-6,8$ & 1 & 1,3 & $0,2-9,4$ & 5 & 4,3 & $1,7-10,7$ \\
\hline Cigarro enrolado à mão & 5 & 2,3 & $1,1-4,7$ & 3 & 3,4 & $1,3-8,7$ & 2 & 1,5 & $0,4-6,0$ \\
\hline Cigarrilha & 2 & 0,8 & $0,1-5,9$ & 0 & - & & 2 & 1,5 & $0,1-9,3$ \\
\hline Charuto, charuto pequeno & 0 & - & & 0 & - & & 0 & - & \\
\hline Narguilé & 188 & 93,3 & $89,9-95,9$ & 81 & 95,3 & $90,1-97,8$ & 107 & 91,7 & $84,6-95,6$ \\
\hline Cigarro indiano/Bidi & 1 & 0,6 & $0,1-4,5$ & 0 & - & & 1 & 1,0 & $0,1-8,1$ \\
\hline \multicolumn{10}{|l|}{ Vitória } \\
\hline $\begin{array}{l}\text { Prevalência de consumo de outros produtos do } \\
\text { tabaco fumado }\end{array}$ & 47 & 4,3 & $3,1-5,7$ & 22 & 4,3 & $3,0-6,1$ & 25 & 4,2 & $2,6-6,8$ \\
\hline \multicolumn{10}{|l|}{ Distribuição de outros produtos de tabaco } \\
\hline \multicolumn{10}{|l|}{ fumado usados com maior frequência ** } \\
\hline Cigarro de cravo/Bali & 4 & 24,6 & $4,8-67,9$ & 2 & 23,9 & $3,6-72,7$ & 2 & 25,4 & $2,6-81,4$ \\
\hline Cigarro enrolado à mão & 0 & - & & 0 & - & & 0 & - & \\
\hline Cigarrilha & 1 & 8,8 & $0,6-61,3$ & 1 & 16,9 & $1,3-75,8$ & 0 & - & \\
\hline Charuto, charuto pequeno & 0 & - & & 0 & - & & 0 & - & \\
\hline Narguilé & 11 & 66,6 & $25,7-92,0$ & 5 & 59,3 & $16,9-91,2$ & 6 & 74,6 & $18,6-97,4$ \\
\hline Cigarro indiano/Bidi & 0 & - & & 0 & - & & 0 & - & \\
\hline
\end{tabular}

* Número de fumantes;

** Após excluir os dados ausentes, o número de indivíduos que responderam a esta pergunta foi inferior a 20; portanto, recomenda-se cautela na interpretação dos resultados.

Tabagismo (PETab) 2 apontaram que a percepção da publicidade pró-tabaco por fumantes jovens nos pontos de venda foi superior à verificada entre os adultos, o que de certa forma sugere que haja um esforço por parte da indústria do tabaco para atingir os adolescentes nas suas ações de promoção e propaganda de produtos do tabaco 4,7,10,11. Esta mesma pesquisa apontou que os jovens residentes no Espírito Santo perceberam duas vezes menos propaganda favorável aos produtos do tabaco do que os jovens residentes em São Paulo ou Mato Grosso do Sul. Logo, novos estudos são necessários para entender a evolução do perfil de consumo dos produtos derivados do tabaco fumado entre os adolescentes estudantes, no cenário nacional, atento às especificidades regionais, tal a diferença observada na prevalência de Vitória em comparação com a das outras duas 
cidades analisadas. Além disso, pelas restrições legislativas de publicidade dos produtos do tabaco 2 , pode haver a utilização ilegal de novas mídias ainda mais próximas dos jovens, tais como propagandas por celulares e Internet, as quais devem ser temas subjacentes de monitoramento contínuo para apoiar o entendimento da expansão do uso de novos produtos 11,12.

Os elevados patamares encontrados de prevalência de consumo de outros produtos de tabaco fumado (principalmente Narguilé) entre os adolescentes das cidades investigadas devem servir de alerta para investir na disseminação ampla da informação e na cessação do consumo de outros produtos do tabaco entre os usuários. Para tanto, é importante direcionar as intervenções de forma a prevenir a aceitação social desse produto e a crença de que eles não são nocivos 10,11,13.

Nota-se que a magnitude de algumas proporções apresentadas deve ser vista com cautela pela baixa precisão das estimativas. Ademais, dado que todas as respostas obtidas foram relatadas diretamente pelos estudantes, i.e., não foram medidas e/ou confirmadas posteriormente, não se pode descartar a possibilidade de ocorrência de viés de informação, quando da interpretação dos resultados.

Segundo dados da PETab, existem hoje no país cerca de 600 mil usuários de outros produtos de tabaco fumado 2 , que podem adquiri-los nos mesmos pontos de venda do cigarro. $\mathrm{O}$ uso de advertências sanitárias nas embalagens de outros produtos de tabaco, nos seus acessórios e/ ou na mídia seria, provavelmente, eficaz para reduzir a proporção de usuários 14. Todavia, ainda há um escasso desenvolvimento de estratégias de comunicação específicas para inibir os seus consumos no Brasil e no mundo 14. Além disso, é necessário dar seguimento às conquistas alcançadas por meio de novas ações, tais como a implementação de lei federal sobre ambientes $100 \%$ livre de todas as formas de tabaco fumado, a proibição total da propaganda de quaisquer produtos de tabaco, inclusive nos pontos de venda, e a busca de alternativas economicamente viáveis para o plantio do fumo, as quais deverão ter os seus impactos avaliados também em pesquisas futuras.

\section{Resumo}

O consumo de outros produtos de tabaco fumado vem crescendo no mundo pelo fato de serem socialmente aceitos e pela crença generalizada de causarem menos dano à saúde do fumante, ajudados também pela globalização das indústrias do tabaco. Recentemente, no Brasil, os profissionais envolvidos com o controle do tabaco atentaram para a importância de monitorar este consumo. Analisaram-se os dados provenientes do Vigescola conduzido, em 2009, em três cidades. As prevalências de uso de outros produtos de tabaco fumado nos últimos 30 dias entre os escolares de 13 a 15 anos foram elevadas em Campo Grande (18,3\%; IC95\%:
14,4\%-22,9\%) e São Paulo (22,1\%; IC95\%: 19,0\%25,6\%). Em Vitória, a prevalência encontrada não foi tão alta quanto às demais (4,3\%; IC95\%: 3,1\%-5,7\%). Não houve diferenças estatisticamente significativas por sexo. Entre os fumantes, o narguilé se destacou por seu alto consumo. É possível que a queda da prevalência de fumantes de cigarro observada nos últimos anos no Brasil tenha favorecido o uso de outros produtos do tabaco como o narguilé, sobretudo entre estudantes.

Tabagismo; Consumo de Produtos Derivados do Tabaco; Prevenção Primária; Vigilância Epidemiológica 


\section{Colaboradores}

A. S. Szklo e M. M. A. Sampaio efetuaram os cálculos e as tabelas, discutiram os resultados e os analisaram junto à equipe. Redigiram o corpo do artigo, tendo trabalhado nele até a sua forma final. E. M. Fernandes participou da coleta dos dados, da discussão dos resultados, tendo colaborado na redação do artigo até a sua forma final. L. M. Almeida coordenou o trabalho da coleta dos dados, participou da discussão dos resultados, tendo colaborado na redação do artigo até a sua forma final.

\section{Agradecimentos}

A todos os técnicos da Divisão de Epidemiologia do Instituto Nacional de Câncer, que auxiliaram na coleta de dados do Vigescola realizado nas cidades de Campo Grande, São Paulo e Vitória, em 2009. Aos coordenadores de curso, funcionários, estudantes das escolas participantes e parceiros internacionais do Global Tobacco Surveillance System (GTSS), que tornaram este estudo possível.

\section{Referências}

1. World Health Organization. Global health risks. Mortality and burden of disease attributable to selected major risks. Geneva: World Health Organization; 2009.

2. Almeida LM, Szklo AS, Souza MC, Sampaio MMA, Mendonça AL, Martins LFL, organizadores. Global addult tobacco survey - Brazil report. Rio de Janeiro: Instituto Nacional de Câncer; 2010.

3. Monteiro CA, Cavalcante T, Moura EC, Claro RM, Szwarcwald CL. Population-based evidence of a strong decline in the prevalence of smokers in Brazil (1989-2003). Bull World Health Organ 2007; 85:527-34.

4. Mejia AB, Ling PM. Tobacco industry consumer research on smokeless tobacco users and product development. Am J Public Health 2010; 100:78-87.

5. Instituto Nacional de Câncer. Vigescola - Vigilância de Tabagismo em Escolares. Dados e fatos de 12 capitais brasileiras. Vol. 1. http://wwwl.inca.gov. $\mathrm{br} /$ tabagismo/31maio2004/vigescola.pdf (acessado em 15/Fev/2011).

6. The Global Youth Tobacco Survey Collaborative Group. Tobacco use among youth: a cross-country comparison. Tob Control 2002; 11:252-70.

7. Warren CW, Jones NR, Eriksen MP. Patterns of global tobacco use in young people and implications for future chronic disease burden in adults. Lancet 2006; 367:749-53.
8. Maziak W, Ward KD, Afifi Soweid RA, Eissenberg T. Tobacco smoking using a waterpipe: a reemerging strain in a global epidemic. Tob Control 2004;13:327-33.

9. WHO Study Group on Tobacco Product Regulation. Waterpipe tobacco smoking: health effects, research needs, and recommended actions by regulators. Geneva: World Health Organization; 2005.

10. Smith-Simone S, Maziak W, Ward KD, Eissenberg T. Waterpipe tobacco smoking: knowledge, attitudes, beliefs, and behavior in two U.S. samples. Nicotine Tob Res 2008; 10:393-8.

11. Damphousse F. Tobacco industry strategies: marketing cigarettes to young people. Promot Educ 2005; Suppl4:30-1,56.

12. World Health Organization. Smoke free movies: from evidence to action. http://whqlibdoc.who. int/publications/2009/9789241597937_eng.pdf (acessado em 30/Jul/2010).

13. Glasgow RE, Gaglio B, France EK, Marcus A, Riley KM, Levinson A, et al. Do behavioral smoking reduction approaches reach more or different smokers? Two studies; similar answers. Addict Behav 2006; 31:509-18.

14. Hammond D. Health warning messages on tobacco products: a review. Tob Control 2011; 20:327-37.

Recebido em 22/Fev/2011

Versão final reapresentada em 04/Jul/2011

Aprovado em 08/Set/2011 